\title{
Pancreatic Carcinoma
}

\section{Weekly Clinical Conference of the Section of Gastroenterology of the University of Michigan*}

\author{
David W. Watson, MD
}

The subject of today's conference is pancreatic tumors, more particularly, pancreatic carcinoma. Each time I am asked to discuss carcinoma of the pancreas at this conference, I review the interval literature and find it distressingly unchanged.

Before presenting the more important features of pancreatic tumors I would like to paraphrase a short quotation by Dr. Warren which I think aptly describes the problem we face in dealing with this organ. Dr. Warren describes the pancreas as a complex and defiant organ, hidden in the upper, deeper recesses of the abdomen where it cannot normally be palpated, intimately associated with the duodenum and biliary system and adjacent to the great abdominal vessels, the stomach and the spleen. It presents an awesome challenge to the surgeon; because it is related physiologically and biochemically to the other organs of digestion, it is important for the maintenance of a healthy, well-nutritioned organism; yet its functions are incompletely understood (1).

Many of us who have dealt with tumors of the pancreas have become discouraged with our inability to effect a great difference in the course these patients pursue from the time they become symptomatic to

From the Department of Medicine, University of Michigan Medical Center, Ann Arbor, Mich.

Address for reprint requests: Dr. D. Watson, Department of Medicine, University of Michigan Medical Center. Ann Arbor, Mich 48104.

* March 16, 1970. their almost inevitable demise. I think there are a number of reasons for this which I hope to bring out along with some faint possibilities for improving our present experience. This is all the more important because of the admittedly poorly documented but prevailing belief that the incidence of carcinoma of the pancreas is increasing.

Figure 1 presents a classification of pancreatic tumors, although the exocrine neoplasms are the ones with which we are concerned today. Endocrine tumors, arising in the islets or elsewhere in the gland, have been the subject of other conferences and will not be discussed at this time. Supporting tissue tumors also occur in the pancreas but are extremely uncommon. The exocrine neoplasms arise either in the acini or are of duct-cell origin and the common,

Fig 1. Classification of pancreatic tumors.

I. Exocrine neoplasms

A. Acinar cells

1. Adenomas (rare)

2. Adenocarcinomas (rare)

B. Duct cells

1. Benign
a. Solid adenoma (rare)
b. Cystadenoma (rare)

2. Malignant

a. Solid carcinoma

(1) Adenocarcinoma (common)

(2) Anaplastic carcinoma (uncommon)

(3) Squamous cell carcinoma (rare)

b. Cystadenocarcinoma (rare)

11. Endocrine neoplasms

III. Supporting tissue tumors 
garden-variety carcinoma of the pancreas is an adenocarcinoma arising from ductular epithelium. Duct-cell neoplasms are usually solid tumors but occasionally may be cystic. These cystadenomas and cystadenocarcinomas, while uncommon, present sufficiently distinctive features (2), differing in their management from either pseudocysts or solid tumors to warrant at least brief consideration. They occur more frequently in females by a ratio of approximately 9 to 1 , whereas solid tumors are more commonly encountered in males. Most patients with cystic tumors are over the age of 50 and present with relatively few symptoms. Such neoplasms are usually encountered as a nontender, palpable mass in the left upper quadrant or the upper, midablomen in a patient with mild, ill-defined abdominal discomfort. It is important to ascertain insofar as possible that the patient has not had pancreatitis or experienced abdominal trauma which would predispose to pseudocyst formation. A rapid change in size of a previously recognized cystic lesion sug. gests that one is more likely dealing with a neoplastic cyst. Surgical exploration should be undertaken in an individual suspected of having a neoplastic cyst, and resection rather than marsupialization carried out. The latter procedure is associated with a significant recurrence rate and is obviously not satisfactory treatment for a cystadenocarcinoma.

Now what about the more common solid tumors of duct-cell origin? These occur twice as frequently in male patients, whose mean age is 55 years. In two-thirds of the cases the head of the pancreas is the site; the body and tail contribute approximately $20 \%$ and $10 \%$, respectively. Three factors have been discussed in the literature as possible predisposing influences. Needless to say, in the majority of patients, such factors are not clearly evident. Some have felt that chronic pancreatitis constitutes one such condition but the evidence is conflicting and highly circumstantial. Diabetes mellitus has been similarly regarded (3), but it is often difficult to know if the abnormality in glucose tolerance has preceded the tumor or has developed as a consequence of it. Indirect support for this view is provided by the observation that ductular hyperplasia is frequent in both diabetics and in patients with pancreatic carcinoma. In some, hyperplasia has been observed to merge with in situ and invasive carcinoma, a situation reminiscent of, but no more enlightening than, that occurring in rectal polyps. A preliminary retrospective study has further suggested that patients who develop carcinoma of the pancreas consume larger amounts of alcohol than those who do not (4). However, the relationship of chronic pancreatitis, heavy alcohol consumption and diabetes mellitus to the origin of carcinoma of the pancreas appears to be tenuous, at best, and for the present must be regarded as unclear.

Everyone is familiar with the classic triad of pain, jaundice and weight loss which represent relatively late symptoms of pancreatic carcinoma, almost always heralding an early death. Ou efforts to recognize early symptoms in these patients are hampered by the fact that only $15 \%$ consult a physician within the first month after the onset of symptoms, and only $65 \%$ do so within 6 months. The importance of this observation is underscored by Sato et al (5) who found that $56 \%$ of carcinomas of the head of the pancreas were resectable when symptoms had been present for less than 3 months, but only $13 \%$, when symptoms existed for a greater length of time.

Many patients initially display rather vague and ill-defined symptoms that either to not occasion personal concern or arouse suspicion in the mind of the plyysician. 
Although painless jaumdice occurring in an older patient is highly suggestive of carcinoma of the pancreas, this tumor, in the majority of instances, is initially characterized by abdominal pain without jaundice. Tumors arising in the head of the pancreas as a rule eventually produce jaundice which, in most instances, is preceded by not more than a few weeks of abdominal pain. Pain, although not always severe, is characteristically persistent or recurrent and progressive. These tumors which tend to infiltrate along nerve trunks and ducts are associated with a desmoplastic response. They tend less to displace nerves, ducts and blood vessels than to envelop and encase them. This feature may account for the severe and unremitting pain these patients eventually experience. Although there are exceptions, the patient who has experienced abdominal pain from a carcinoma arising in the head of the pancreas will in general become jaundiced within 3 months of the onset of pain. For tumors arising in the body or tail, jaundice may never supervene; if it does, it will generally do so after a much longer interval of discomfort. It is worth remembering that pain of pancreatic origin tends to localize in the corresponding area of the abdomen. Thus tumors located in the head will produce more intense pain in the right epigastrium, whereas those located in the body will tend to localize to the midline, and those occurring in the tail may refer to the left upper quadrant. Tumors arising in the tail may be the most difficult to diagnose (6) since they seldom produce jaundice, and usually lead to left upper quadrant or left midabdominal pain which is often not suspected to be of pancreatic origin. Tumors in this location may obstruct the splenic vein, leading to splenomegaly and hypersplenism; may obstruct the portal vein and produce esophageal varices; or may obstruct the splenic artery, resulting in an arterial bruit in the left upper quadrant. Such bruits, however, must be interpreted with caution since they may occur with a very large spleen regardless of cause, arteriovenous fistulas, splenic artery aneurysms and arteritis.

In the jaundiced patient, a palpable gallbladder has been emphasized as signifying extrahepatic biliary obstruction by tumnr rather than calculus. This is not invariably true. Furthemore, many otherwise palpable gallbladders are missed because of failure to search carefully in the proper location. As the gallbladder distends and the liver enlarges as a result of biliary obstruction, the fundus of the gallbladder is diswace I inferiorly and laterally and therefore may be found far removed from its expected location.

A number of less common clinical features associated with pancreatic carcinoma are presented in Fig 2. Hypoglycemia, although a rare manifestation of these tumors, is almost invariably associated with complete obstruction of the main pancreatic duct.

Much has been written concerning the relationship of migratory thrombophlebitis

Fig 2. Unusual manifestations of carcinoma of the pancreas.

Hypoglycemia $(7)$
Cardiovascular
Venous thromboses
Arterial embolization
Hypercalcemia $(8,9)$
Psychiatric changes $(10)$
Depression with crying spells
Intractable insomnia
Anxiety
Premonition of serious illness
Carcinoid syndrome
Subcutaneous fat necrosis (11)
Portal hypertension
Splenomegaly and hypersplenism
Left upper quadrant arterial bruit
Inappropriate ADH secretion


and pancreatic carcinoma. Such an association is probably not novel but is likely to be a feature of many intra-abdominal malignancies resulting in debilitation and prolonged bed rest. The same interpretation can be applied to so-called marantic endocarditis and arterial embolization. $\mathrm{Hy}$ percalcemia has been reported with ductcell carcinomas but it is not entirely clear that such tumors are not actually of isletcell origin or carcinoid in type. Similarly, the carcinoid syndrome, although ascribed to some cases of duct-cell carcinoma, probably occurs only with carcinoid tumors which have arisen in the pancreas.

Psychiatric changes are difficult to characterize and quantitate but appear to occur more frequently in association with carcinoma of the pancreas than other malignant lesions of the digestive tract. When associated with otherwise vague abdominal symptoms, these changes should direct attention to this organ.

Subcutaneous fat necrosis, appearing as tender, subcutaneous nodules originating on the lower extremities and later involv. ing other portions of the body, is accompanied by polyarthralgia and a modest peripheral eosinophilia. Although uncommon, such lesions have been found only with the rarer acinar-cell carcinomas. These tumors have an extremely high lipase content which in some way is probably responsible.

Unfortunately, the clinical laboratory has been of limited usefulness in the diagnosis of carcinoma of the pancreas. The most frequently observed abnormality is a decrease in glucose tolerance which occurs in from 20 to $50 \%$ of cases. This will be most readily recognized if a glucose tolerance test is carried out, rather than depending on glucosuria or fasting hyperglycemia. The cause remains obscure and cannot be related to extent of replacement of the normal pancreas by tumor. 'The recent appearance of diabetes in a patient without a genetic predisposition, when accompanied by abdominal complaints, should call for a thorough investigation. An unexplained increase in insulin requirement in an established diabetic with gastrointestinal symptoms should be similarly regarded.

A few patients may exhibit minor rises in serum or, more particularly, in urinary amylase values. Such changes are probably related to ductal obstruction and associated pancreatitis and have little diagnostic value.

The use of pancreozymin-secretin preparations to measure pancreatic reserve may have some value in localizing otherwise vague abdominal symptoms to the pancreas without revealing their exact cause. The differentiation between pancreatitis and carcinoma, while sometimes possible, is often imprecise. In some hands the concurrent examination of duodenal contents for maïignant cells has been helpful (13), positive results varying between 20 and $70 \%$ in proven cases. For reasons that are not clear to me, we have never been particularly successful with this technic in our own laboratory. Much depends on the competence of the cytologist but even more on the facility with which the specimens are collected and processed.

Pancreatic scanning technics are relatively recent additions to our diagnostic approach but tend to fall heir to the same limitations attending secretory tests, namely, the frequent inability to differentiate tumor from areas of pancreatitis and cyst formation.

This brings us to a consideration of radiographic procedures which, I think, currently constitute the most useful diagnostic approach to pancreatic neoplasms. The three technics which are applicable include barium contrast examination of 
the stomach and duodenum, selective arteriography and percutaneous cholangiography. In regard to the first of these, we are not interested in the widened and grossly distorted duodenal loop classically associated with carcinoma of the head of the pancreas, which almost invariably signifies an incurable if not inoperable lesion. Rather, we are interested in small areas of nodularity along the medial aspect of the duodenal sweep, or distortion and effacement of the duodenal folds in this region. The ability to detect relatively minor changes of this type may be enhanced by the use of hypotonic duodenography. One must be cognizant, however, of the fact that similar changes in the duodenogram may be prom duced by chronic pancreatitis or collateral circulation associated with portal hyperten. sion from other causes.

Of more value, I think, in defining pancreatic lesions including carcinomas is selective arteriography. The incidence of angiographic abnormalities in proved cases ranges from $90 \%$ in a retrospective analysis by Lunderquist to $29 \%$ in a prospective analysis by Nebesar and Pollard. The experience of Bookstein, Reuter and Martel (14) at the University of Michigan and Wayne County Hospital is, however, generally encouraging. Prospective diagnoses were correct in $75 \%$ of carcinoma cases, in none of which was a completely normal arteriogram present. False positives occurred at a rate of $3 \%$. Chronic pancreatitis offers the most difficulty for the angiog. rapher, and in this group alone the false positive rate is likely to be highest. The authors indicate that irregular encasement, pathologic vessels and occlusion of large arteries are the most reliable signs of carcinoma. They further point out that optimal technic is vital, and superselective injections are often indispensable.

One might imagine that such angiograph- ic abnormalities would be associated with a higher incidence of metastases, thereby heralding a more dismal prognosis and providing no really useful information. This possibility remains undetermined although the experience of Sato et al (5) points to a high likelihood of nonresectability when abnormalities of the common hepatic or gastroduodenal artery are demonstrated.

For the jaundiced patient in whom the responsible lesion cannot be localized to an intra- or extrahepatic site, percutaneous cholangiography may be helpful. The patient must be scheduled for celiotomy, since, in the event a dilated ductal system is found, exploration and relief of the obstruction are imperative. If an extrahepatic obstruction cannot be demonstrated, the patient is returned to his bed and a percutaneous liver biopsy is performed. While not a so-called routine procedure, it may be of considerable usefulness to the patient in whom the differentiation lies between extrahepatic obstruction (as from a pancreatic tumor) and intrahepatic cholestasis (especially that which is drug-induced).

Confirmation of the diagnosis of carcinoma of the pancreas remains surgical. When resection is carried out, the pathologist will of course provide a definite answer in the majority of instances. In the absence of resection, the internist must depend upon the visual and tactile skills of the surgeon for an impression. Quite naturally, this will vary widely. Biopsy has generally been discouraged on the grounds that it will either result in tumor spread or predispose to a high frequency of serious complications. Neither view is as well founded as many of us have been led to believe (15); perhaps biopsy should be undertaken more often when the gross impression is doubtful and resection is not contemplated.

Although treatment of pancreatic carcinoma continues to be unsatisfactory, an 
additional reason for undertaking a vigorous diagnostic approach is the possibility that some patients suspected of having carcinoma of the pancreas may, in fact, have periampullary tumors possessing a far better prognosis. Tumors arising in the ampulla, distal bile duct or duodenum have a 5 year survival of $35-40 \%$, about $4-5$ times that for carcinoma of the pancreas.

Like the final diagnosis, treatment remains largely the province of the surgeon. Unfortunately, the overall resectability rate for carcinomas of the head does not exceed $25 \%$ at best, and is even lower for tumors of the body and tail. The 5-year survival for all tumors whether resectable or not is probably less than $1 \%$. For those in whom a pancreaticoduodenectomy is possible, this figure becomes $7 \%$ (16). This procedure is generally regarded as carrying a mortality of at least $10 \%$ although Warren et al have reported a figure of $2.9 \%$ (1). One might logically wonder, therefore, about the benefits to be derived from operative intervention in these patients. Apart from the possibility that a small number of patients can, in fact, be cured, and other more readily treatable periampullary tumors discovered, significant palliation through the relief of pruritus or vomiting is a worthwhile objective. Unfortunately, the mean survival after radical surgery is 15 months, again emphasizing our inability to prolong life in the majority of individuals with this lesion.

In this discussion I have endeavored to point out some of the more useful and unusual clinical features of carcinoma of the pancreas, as well as the diagnostic procedures currently available. It must be emphasized that all diagnostic studies, while of assistance in proving that the patient has a pancreatic carcinoma, are of little or no help in establishing this early enough to improve prognosis. At present, I think we must content ourselves with exer- cising a keen index of suspicion in patients with vague upper abdominal symptoms, particularly when associated with some of the more subtle and umusual features discussed. A more liberal and prompt recourse to hypotonic duodenography and selective arteriogxaphy, while not guaranteeing a significant change in prognosis, may result in an increased likelihood of cure in some individuals. Although not assuring a more favorable outcome, earlier diagnosis must derive from an emphasis on early and minimal symptoms and less restricted recourse to abdominal exploration.

Dr. Pinedo: From what you said, I gather that there is no diagnostic procelure that can differentiate between pancreatitis and carcinoma with certainty.

Dr. Watson: In general, that is true. The definitive diagnosis is most frequently made after laparotony. Selective arteriography, particularly with further experience, may prove a welcome exception, however. Unfortunately the more informative the evidence, the more advanced the tumor and the less chance of cure. Early surgical exploration is to be emphasized.

Dr. Kuiper: In those patients who are inoperable, would you use a chemotherapeutic agent?

Dr. Watson: Present experience indicates that chemotherapy has relatively little or nothing to offer the patient with inoperable pancreatic carcinoma either in terms of increased survival or palliation. In respect to pain, we have been impressed by the ability of salicylates or Darvon to provide relief in excess of that which might be expected on the basis of narcotic requirements. The regular use of these medications is often helpful in reducing or even eliminating the neel for narcotics.

Dr. Kaufman: I just want to ask you whether you nould regard the presence of back pain as an index of inoperability since 
this might imply involvement of posterior somatic nerve roots.

Dr. Watson: I would have to say no. Impingement rather than infiltration might produce pain.

Dr. Pinedo: There are a significant number of patients with abdominal pain who have normal barium studies and in whom it is not possible to arrive at a diagnosis of pancreatic disease. Do you think we should do arteriograms in all such patients?

Dr. Watsom: Certainly clinical judgement must be exercised in ordering arteriograms. I believe they should be utilized more extensively than is presently the case, especially in patients exhibiting one or more of the features previously discussed.

Well, our time is up but I think our discussion today has at least emphasized the problems we face in trying to deal with pancreatic neoplasms, and I hope it has provided some perspective which may be useful in their diagnosis and management.

\section{REFERENCES}

1. Warren KW, Brasch JW, Thum CW: Carcinoma of the pancreas. Surg Clin N Amer 48:601-618, 1968

2. Becker WF, Welsh RI. Pratt HS: Cystadenoma and cystadenocarcinoma of the pancreas. Ann Surg 161:845-863, 1965

3. Karmody AJ. Kyle J: The association between carcinoma of the pancreas and diabetes mellitus. Brit J Surg 56:362-364, 1969

4. Burch GE, Ansari A: Chronic alcoholism and carcinoma of the pancreas. Arch Intern Ifed (Chicago) 122:273-275, 1968

5. Sato T', Saitoh Y, Koyama K, et al: Preop- erative determination of operability in carcinomas of the pancreas and the periampullary region. Ann Surg 168:876-886, 1968

6. Arlen M, Brockunier A Jr: Clinical manifestations of carcinoma of the tail of the pancreas. Cancer 20:1920-1923, 1967

7. IcBce JW, Lanza FL, Erickson EE: Hypoglycemia due to obstruction of pancreatic excretory ducts by carcinoma. Arch path $81: 287-291,1966$

8. Marks C: Hypercalcenia in malignant nonparathyroid disease. Amer Surg 31:254-258, 1965

9. Snedecor P.A, Baker HW: Pseudohyperparathyroidism due to malignant tumors. Cancer 17:1492-1496, 1964

10. Fras I, Litin F.M, Pearon Js: Comparison of psychiatric symptoms in carcinoma of the pancreas with those in some other intraabdominal neoplasms. Amer J Psychiat 123: $1553-1562,1967$

11. Naciathon Hl: Brown P.l, Shen EM: Acinar cell carcinoma of the pancreas with subcutaneous fat necrosis. Gastroenterology 49:555-559, 1965

12. Marks LJ, Berde B. Klein LA. et al: Mappropriate vasopressin secretion and carcinoma of the pancreas. Amer J Med 45: 967-974, 1968

13. Goldstein H. Ventrke L.k: Value of exfoliative cytology in pancreatic carcinoma. Gut 9:316-318, 1968

14. Bookstein JJ, Reuter SR, Martel W: Angiographic evaluation of pancreatic carcinoma. Radiology 93:757-764, 1969

15. Forsgren L, Goran L, Nordenstam H: Pancreatic biopsy. Acta Chir Scand 134:457460, 1968

16. Elias EG: Carcinoma of the pancreas. Arch Surg 98:138-140, 1969 